\title{
The characteristic equation of the exceptional Jordan algebra: its eigenvalues, and their possible connection with mass ratios of quarks and leptons
}

\author{
Tejinder P. Singh \\ Tata Institute of Fundamental Research, Homi Bhabha Road, Mumbai 400005, India \\ e-mail: tpsingh@tifr.res.in \\ v1 Submitted for publication to Adv. App. Clifford Algebras on Dec. 29, 2020 \\ v2, January 6, 2021 Remarks on fine structure constant added on p. 21 \\ This versioin v3. January 22, 2021: Added: \\ 'Karolyhazy correction to the asymptotic fine structure constant', $p .24$
}

\begin{abstract}
The exceptional Jordan algebra [also known as the Albert algebra] is the finite dimensional algebra of $3 \times 3$ Hermitean matrices with octonionic entries. Its automorphism group is the exceptional Lie group $F_{4}$. These matrices admit a cubic characteristic equation whose eigenvalues are real and depend on the invariant trace, determinant, and an inner product made from the Jordan matrix. Also, there is some evidence in the literature that the group $F_{4}$ could play a role in the unification of the standard model symmetries, including the Lorentz symmetry. The octonion algebra is known to correctly yield the electric charge values $(0$, $1 / 3,2 / 3,1)$ for standard model fermions, via the eigenvalues of a $U(1)$ number operator, identified with $U(1)_{\mathrm{em}}$. In the present article, we use the same octonionic representation of the fermions to compute the eigenvalues of the characteristic equation of the Albert algebra, and compare the resulting eigenvalues with the known mass ratios for quarks and leptons. We find that the ratios of the eigenvalues correctly reproduce the [square root of the] known mass ratios for up, charm and top quark. We also propose a diagrammatic representation of the standard model bosons, Higgs and three fermion generations, in terms of the octonions, exhibiting an $F_{4}$ symmetry. We motivate from our Lagrangian as to why the eigenvalues computed in this work could bear a relation with mass ratios of quarks and leptons. In conjunction with the trace dynamics Lagrangian, the Jordan eigenvalues also provide a first principles theoretical derivation of the low energy value of the fine structure
\end{abstract}


constant, yielding the value 1/137.04006. The Karolyhazy correction to this value gives an exact match with the measured value of the constant, after assuming a specific value for the electro-weak symmetry breaking energy scale.

\section{INTRODUCTION}

The possible connection between division algebras, exceptional Lie groups, and the standard model has been a subject of interest for many researchers in the last few decades [1-23]. Our own interest in this connection stems from the following observation [24]. In the pre-geometric, pre-quantum theory of generalised trace dynamics, the definition of spin requires $4 \mathrm{D}$ space-time to be generalised to an 8D non-commutative space. In this case, an octonionic space is a possible, natural, choice for further investigation. We found that the additional four directions can serve as 'internal' directions and open a path towards a possible unification of the Lorentz symmetry with the standard model, with gravitation arising only as an emergent phenomenon. Instead of the Lorentz transformations and internal gauge transformations, the symmetries of the octonionic space are now described by the automorphisms of the octonion algebra. Remarkably enough, the symmetry groups of this algebra, namely the exceptional Lie groups, naturally have in them the desired symmetries [and only those symmetries, or higher ones built from them] of the standard model, including Lorentz symmetry, without the need for any fine tuning or adjustments. Thus the group of automorphisms of the octonions is $G_{2}$, the smallest of the five exceptional Lie groups $G_{2}, F_{4}, E_{6}, E_{7}, E_{8}$. The group $G_{2}$ has two intersecting maximal sub-groups, $S U(3) X U(1)$ and $S U(2) X S U(2)$, which between them account for the fourteen generators of $G_{2}$, and can possibly serve as the symmetry group for one generation of standard model fermions. The complexified Clifford algebra $C l(6, C)$ plays a very important role in establishing this connection. In particular, motivated by a map between the complexified octonion algebra and $C l(6, C)$, electric charge is defined as one-third the eigenvalue of a $U(1)$ number operator, which is identified with $U(1)_{e m}[3,5]$.

Describing the symmetries $S U(3) X U(1)$ and $S U(2) X S U(2)$ of the standard model [with Lorentz symmetry now included] requires two copies of the Clifford algebra $C l(6, C)$ whereas the octonion algebra yields only one such independent copy. It turns out that if boundary terms are not dropped from the Lagrangian of our theory, the Lagrangian describes three 
fermion generations, with the symmetry group now raised to $F_{4}$. This admits three intersecting copies of $G_{2}$, with the $S U(2) X S U(2)$ in the intersection, and a Clifford algebra construction based on the three copies of the octonion algebra is now possible [25]. Attention thus shifts to investigating the connection between $F_{4}$ and the three generations of the standard model.

$F_{4}$ is also the group of automorphisms of the exceptional Jordan algebra [11, 26, 27]. The elements of the algebra are $3 \times 3$ Hermitean matrices with octonionic entries. This algebra admits an important cubic characteristic equation with real eigenvalues. Now we know that the three fermion generations differ from each other only in the mass of the corresponding fermion, whereas the electric charge remains unchanged across the generations. This motivates us to ask: if the eigenvalues of the $U(1)$ number operator constructed from the octonion algebra represent electric charge, what is represented by the eigenvalues of the exceptional Jordan algebra? Could these eigenvalues bear a connection with mass ratios of quarks and leptons? This is the question investigated in the present paper. Using the very same octonion algebra which was used to construct a state basis for standard model fermions, we calculate these eigenvalues. Remarkably, the eigenvalues are very simple to express, and bear a simple relation with electric charge. We comment on how they could relate to mass ratios. In particular we find that the ratios of the eigenvalues match with the square root of the mass ratios of up quark, charm, and the top. [These eigenvalues are invariant under algebra automorphisms.]

Subsequently in the paper we propose a diagrammatic representation, based on octonions and $F_{4}$, of the fourteen gauge bosons, and the $(8 \times 2) \times 3=48$ fermions of three generations of standard model, along with the four Higgs. We attempt to explain why there are not three generations of bosons, and re-express our Lagrangian in a form which explicitly reflects this fact. We hint at how this Lagrangian might directly lead to the characteristic equation of the exceptional Jordan algebra, and reveal why the eigenvalues might be related to mass. 


\section{EIGENVALUES FROM THE CHARACTERISTIC EQUATION OF THE EX- CEPTIONAL JORDAN ALGEBRA}

The exceptional Jordan algebra $[\mathrm{EJA}] J_{3}(\mathbb{O})$ is the algebra of $3 \times 3$ Hermitean matrices with octonionic entries $[12,21,22,26]$

$$
X(\xi, x)=\left[\begin{array}{lll}
\xi_{1} & x_{3} & x_{2}^{*} \\
x_{3}^{*} & \xi_{2} & x_{1} \\
x_{2} & x_{1}^{*} & \xi_{3}
\end{array}\right]
$$

It satisfies the characteristic equation [12, 21, 22]

$$
X^{3}-\operatorname{Tr}(X) X^{2}+S(X) X-\operatorname{Det}(X)=0 ; \quad \operatorname{Tr}(X)=\xi_{1}+\xi_{2}+\xi_{3}
$$

which is also satisfied by the eigenvalues $\lambda$ of this matrix

$$
\lambda^{3}-\operatorname{Tr}(X) \lambda^{2}+S(X) \lambda-\operatorname{Det}(X)=0
$$

Here the determinant is

$$
\operatorname{Det}(X)=\xi_{1} \xi_{2} \xi_{3}+2 R e\left(x_{1} x_{2} x_{3}\right)-\sum_{1}^{3} \xi_{i} x_{i} x_{i}^{*}
$$

and $S(X)$ is given by

$$
S(X)=\xi_{1} \xi_{2}-x_{3} x_{3}^{*}+\xi_{2} \xi_{3}-x_{1} x_{1}^{*}+\xi_{1} \xi_{3}-x_{2}^{*} x_{2}
$$

The diagonal entries are real numbers and the off-diagonal entries are (real-valued) octonions. A star denotes an octonionic conjugate. The automorphism group of this algebra is the exceptional Lie group $F_{4}$. Because the Jordan matrix is Hermitean, it has real eigenvalues which can be obtained by solving the above-given eigenvalue equation.

In the present Letter we suggest that these eigenvalues carry information about mass ratios of quarks and leptons of the standard model, provided we suitably employ the octonionic entries and the diagonal real elements to describe quarks and leptons of the standard 
model. Building on earlier work $[3,4,19]$ we recently showed that the complexified Clifford algebra $C l(6, C)$ made from the octonions acting on themselves can be used to obtain an explicit octonionic representation for a single generation of eight quarks and leptons, and their anti-particles. In a specific basis, using the neutrino as the idempotent $V$, this representation is as follows [3, 24]. The $\alpha$ are fermionic ladder operators of $C l(6, C)$ (please see Eqn. (34) of [24]).

$$
\begin{array}{rrr}
V=\frac{i}{2} e_{7} & {\left[V_{\nu}\right. \text { Neutrino] }} \\
\alpha_{1}^{\dagger} V=\frac{1}{2}\left(e_{5}+i e_{4}\right) \times V=\frac{1}{4}\left(e_{5}+i e_{4}\right) \quad\left[\mathrm{V}_{\mathrm{ad} 1} \text { Anti }- \text { down quark }\right] \\
\alpha_{2}^{\dagger} V=\frac{1}{2}\left(e_{3}+i e_{1}\right) \times V=\frac{1}{4}\left(e_{3}+i e_{1}\right) \quad\left[\mathrm{V}_{\mathrm{ad} 2} \text { Anti }- \text { down quark }\right] \\
\alpha_{3}^{\dagger} V=\frac{1}{2}\left(e_{6}+i e_{2}\right) \times V=\frac{1}{4}\left(e_{6}+i e_{2}\right) \quad\left[\mathrm{V}_{\mathrm{ad} 3} \text { Anti }-\right. \text { down quark] } \\
\alpha_{3}^{\dagger} \alpha_{2}^{\dagger} V=\frac{1}{4}\left(e_{4}+i e_{5}\right) & {\left[\mathrm{V}_{\mathrm{u} 1}\right. \text { Up quark] }} \\
\alpha_{1}^{\dagger} \alpha_{3}^{\dagger} V==\frac{1}{4}\left(e_{1}+i e_{3}\right) & {\left[\mathrm{V}_{\mathrm{u} 2}\right. \text { Up quark] }} \\
\alpha_{2}^{\dagger} \alpha_{1}^{\dagger} V=\frac{1}{4}\left(e_{2}+i e_{6}\right) & {\left[\mathrm{V}_{\mathrm{u} 3}\right. \text { Up quark] }} \\
\alpha_{3}^{\dagger} \alpha_{2}^{\dagger} \alpha_{1}^{\dagger} V=-\frac{1}{8}\left(i+e_{7}\right) & {\left[\mathrm{V}_{\mathrm{e}+}\right. \text { Positron] }}
\end{array}
$$

The anti-particles are obtained from the above representation by complex conjugation [3].

In the context of the projective geometry of the octonionic projective plane $\mathbb{O} P^{2}$ it has been shown by Baez [14] that upto automorphisms, projections in EJA take one of the following four forms, having the respective invariant trace $0,1,2,3$.

$$
\begin{gathered}
p_{0}=\left[\begin{array}{lll}
0 & 0 & 0 \\
0 & 0 & 0 \\
0 & 0 & 0
\end{array}\right] \\
p_{1}=\left[\begin{array}{lll}
1 & 0 & 0 \\
0 & 0 & 0 \\
0 & 0 & 0
\end{array}\right]
\end{gathered}
$$




$$
\begin{aligned}
& p_{2}=\left[\begin{array}{lll}
1 & 0 & 0 \\
0 & 1 & 0 \\
0 & 0 & 0
\end{array}\right] \\
& p_{3}=\left[\begin{array}{lll}
1 & 0 & 0 \\
0 & 1 & 0 \\
0 & 0 & 1
\end{array}\right]
\end{aligned}
$$

Since it has earlier been shown by Furey [3] that electric charge is defined in the division algebra framework as one-third of the eigenvalue of a $U(1)$ number operator made from the generators of the $S U(3)$ in $G_{2}$, we propose to identify the trace of the Jordan matrix with the sum of the charges of the three identically charged fermions across the three generations. Thus the trace zero Jordan matrix will have diagonal entries zero, and will represent the (neutrino, muon neutrino, tau-neutrino). The trace one Jordan matrix will have diagonal entries $(1 / 3,1 / 3,1 / 3)$ and will represent the (anti-down quark, anti-strange quark, anti-bottom quark). [Color is not relevant for determination of mass eigenvalues, and hence effectively we have four fermions per generation: two leptons and two quarks, after suppressing color]. The trace two Jordan matrix will have entries $(2 / 3,2 / 3,2 / 3)$ and will represent the (up quark, charm, top). Lastly, the trace three Jordan matrix will have entries $(1,1,1)$ and will represent (positron, anti-muon, anti-tau-lepton).

We have thus identified the diagonal real entries of the four Jordan matrices whose eigenvalues we seek. We must next specify the octonionic entries in each of the four Jordan matrices. Note however that the above representation of the fermions of one generation is using complex octonions, whereas the entries in the Jordan matrices are real octonions. So we devise the following scheme for a one-to-one map from the complex octonion to a real octonion. Since we are ignoring color, we pick one out of the three up quarks, say $\left(e_{4}+i e_{5}\right)$, and one of three anti-down quarks, say $\left(e_{5}+i e_{4}\right)$. Since the representation for the electron and the neutrino use $e_{7}$ and a complex number, it follows that the four octonions we have picked form the quaternionic triplet $\left(e_{4}, e_{5}, e_{7}\right)$ [we use the Fano plane convention shown in the figure below]. Hence the four said octonions are in fact complex quaternions, thus belonging to the general form

$$
\left(a_{0}+i a_{1}\right)+\left(a_{2}+i a_{3}\right) e_{4}+\left(a_{4}+i a_{5}\right) e_{5}+\left(a_{6}+i a_{7}\right) e_{7}
$$


where the eight $a$-s are real numbers. By definition, we map this complex quaternion to the following real octonion:

$$
a_{0}+a_{1} e_{1}+a_{5} e_{2}+a_{3} e_{3}+a_{2} e_{4}+a_{4} e_{5}+a_{7} e_{6}+a_{6} e_{7}
$$

Note that the four real coefficients in the original complex quaternion have been kept in place, and their four imaginary counterparts have been moved to the octonion directions $\left(e_{1}, e_{2}, e_{3}, e_{6}\right)$ now as real numbers. Clearly, the map is reversible, given the real octonion we can construct the equivalent complex quaternion representing the fermion. We can now

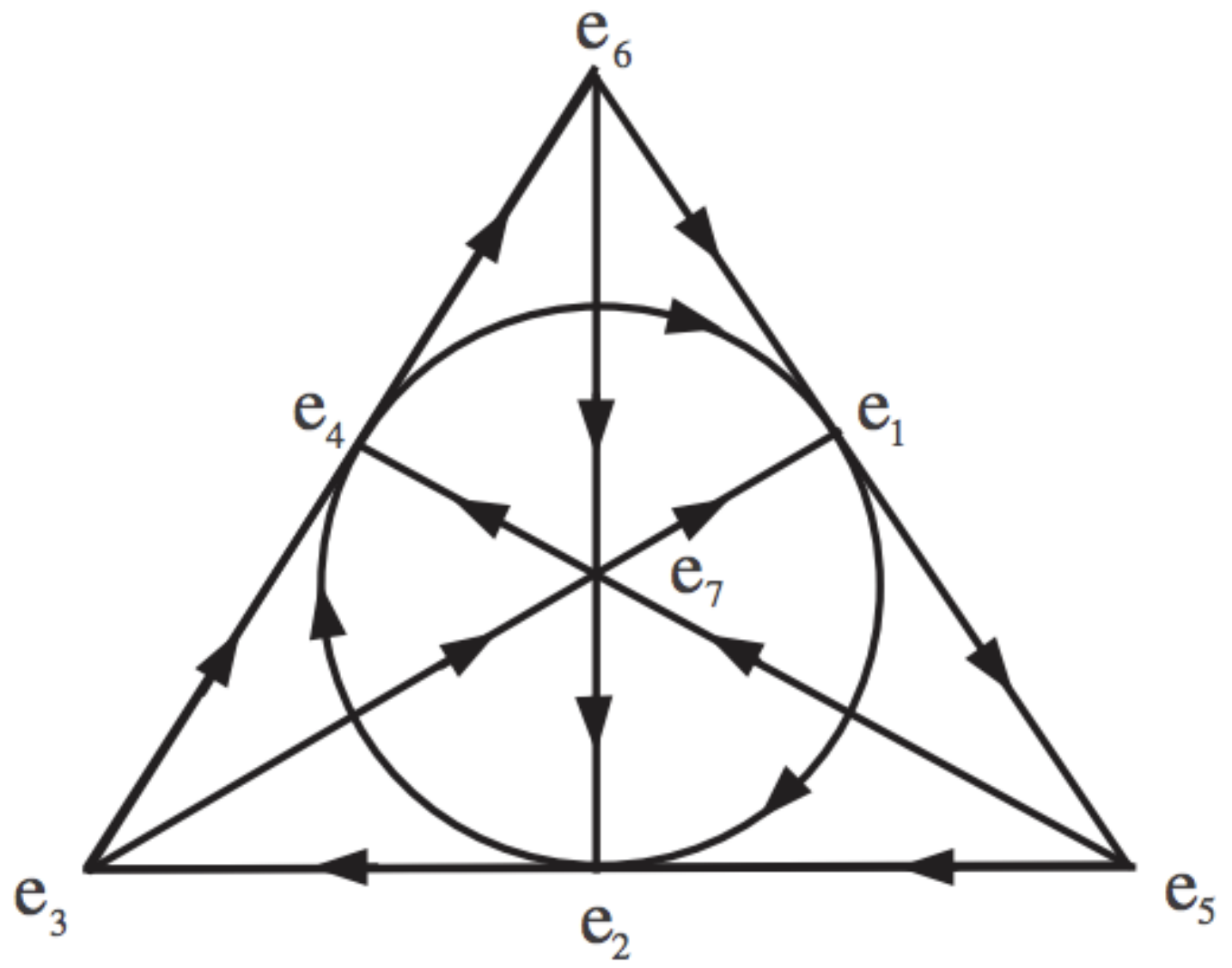

FIG. 1. The Fano plane.

use this map and construct the following four real octonions for the neutrino, anti-down quark, up quark and the positron, respectively, after comparing with their complex octonion 
representation above.

$$
\begin{gathered}
V_{\nu}=\frac{i}{2} e_{7} \longrightarrow \frac{1}{2} e_{6} \\
V_{a d}=\frac{1}{4} e_{5}+\frac{i}{4} e_{4} \longrightarrow \frac{1}{4} e_{5}+\frac{1}{4} e_{3} \\
V_{u}=\frac{1}{4} e_{4}+\frac{i}{4} e_{5} \longrightarrow \frac{1}{4} e_{4}+\frac{1}{4} e_{2} \\
V_{e^{+}}=-\frac{i}{8}-\frac{1}{8} e_{7} \longrightarrow-\frac{1}{8}-\frac{1}{8} e_{7}
\end{gathered}
$$

These four real octonions will go, one each, in the four different Jordan matrices whose eigenvalues we wish to calculate. Next, we need the real octonionic representations for the four fermions [color suppressed] in the second generation and the four in the third generation. We propose to build these as follows, from the real octonion representations made just above for the first generation. Since $F_{4}$ has the inclusion $S U(3) \times S U(3)$, one $\left.S U 3\right)$ being for color and the other for generation, we propose to obtain the second generation by a $2 \pi / 3$ rotation on the first generation, and the third generation by a $2 \pi / 3$ rotation on the second generation. By this we mean the following construction, for the four respective Jordan matrices:

Up quark / Charm / Top: The up quark is $\left(e_{4} / 4+e_{2} / 4\right)$ We think of this as a 'plane' and rotate this octonion by $2 \pi / 3$ by left multiplying it by $e^{2 \pi e_{2} / 3}=-1 / 2+\sqrt{3} e_{2} / 2$. This will be the charm quark $V_{c}$. Then we left multiply the charm quark by $e^{2 \pi e_{2}} / 3$ to get the top quark $V_{t}$. Hence we have,

$V_{c}=\left(-1 / 2+\sqrt{3} e_{2} / 2\right) \times V_{u}=\left(-1 / 2+\sqrt{3} e_{2} / 2\right) \times\left(\frac{1}{4} e_{4}+\frac{1}{4} e_{2}\right)=-\frac{1}{8} e_{4}-\frac{1}{8} e_{2}-\frac{\sqrt{3}}{8}-\frac{\sqrt{3}}{8} e_{1}$

We have used the conventional multiplication rules for the octonions, which are reproduced below in Fig. 2, for ready reference. Similarly, we can construct the top quark by a $2 \pi / 3$ rotation on the charm:

$$
\begin{aligned}
V_{t} & =\left(-1 / 2+\sqrt{3} e_{2} / 2\right) \times V_{c}=\left(-1 / 2+\sqrt{3} e_{2} / 2\right) \times\left(-\frac{1}{8} e_{4}-\frac{1}{8} e_{2}-\frac{\sqrt{3}}{8}-\frac{\sqrt{3}}{8} e_{1}\right) \\
& =-\frac{1}{8} e_{4}-\frac{1}{8} e_{2}+\frac{\sqrt{3}}{8}+\frac{\sqrt{3}}{8} e_{1}
\end{aligned}
$$

Next, we construct the anti-strange $V_{a s}$ and anti-bottom $V_{a b}$, by left-multiplication of the 


\begin{tabular}{|c|c|c|c|c|c|c|c|c|}
\hline & $\mathrm{e} 0$ & $\mathrm{e} 1$ & e2 & e4 & e3 & e5 & e6 & e7 \\
\hline e0 & 1 & e1 & e2 & e4 & e3 & e5 & e6 & e7 \\
\hline e1 & e1 & -1 & e4 & -e2 & e7 & e6 & $-\mathrm{e} 5$ & $-\mathrm{e} 3$ \\
\hline e2 & e2 & $-\mathrm{e} 4$ & -1 & e1 & e5 & $-\mathrm{e} 3$ & e7 & $-e 6$ \\
\hline e4 & e4 & $\mathrm{e} 2$ & $-\mathrm{e} 1$ & -1 & -e6 & e7 & e3 & -e5 \\
\hline e3 & e3 & -e7 & -e5 & e6 & -1 & e2 & $-\mathrm{e} 4$ & e1 \\
\hline e5 & e5 & -e6 & e3 & -e7 & $-\mathrm{e} 2$ & -1 & e1 & e4 \\
\hline e6 & e6 & e5 & -e7 & -e3 & e4 & -e1 & -1 & e2 \\
\hline e7 & e7 & e3 & e6 & e5 & $-e 1$ & -e4 & $-e 2$ & -1 \\
\hline
\end{tabular}

FIG. 2. The multiplication table for two octonions. Elements in the first column on the left, left multiply elements in the top row.

anti-down quark $V_{a d}$ by $e^{2 \pi e_{3} / 3}$.

$$
\begin{gathered}
V_{a s}=\left(-\frac{1}{2}+\frac{\sqrt{3}}{2} e_{3}\right) \times V_{a d}=\left(-\frac{1}{2}+\frac{\sqrt{3}}{2} e_{3}\right) \times\left(\frac{1}{4} e_{5}+\frac{1}{4} e_{3}\right) \\
=-\frac{1}{8} e_{5}-\frac{1}{8} e_{3}+\frac{\sqrt{3}}{8} e_{2}-\frac{\sqrt{3}}{8} \\
V_{a b}=\left(-\frac{1}{2}+\frac{\sqrt{3}}{2} e_{3}\right)\left(-\frac{1}{8} e_{5}-\frac{1}{8} e_{3}+\frac{\sqrt{3}}{8} e_{2}-\frac{\sqrt{3}}{8}\right) \\
=-\frac{1}{8} e_{5}-\frac{\sqrt{3}}{8} e_{2}-\frac{1}{8} e_{3}+\frac{\sqrt{3}}{8}
\end{gathered}
$$

Next, we construct the octonions for the anti-muon $V_{a \mu}$ and anti-tau-lepton $V_{a \tau}$ by left 
multiplying the positron $V_{e^{+}}$by $e^{2 \pi e_{1} / 3}$

$$
\begin{gathered}
V_{a \mu}=\left(-\frac{1}{2}+\frac{\sqrt{3}}{2} e_{1}\right) \times\left(-\frac{1}{8} e_{1}-\frac{1}{8} e_{7}\right) \\
=\frac{1}{16} e_{1}+\frac{1}{16} e_{7}+\frac{\sqrt{3}}{16}+\frac{\sqrt{3}}{16} e_{3} \\
V_{a \tau}=\left(-\frac{1}{2}+\frac{\sqrt{3}}{2} e_{1}\right) \times\left(\frac{1}{16} e_{1}+\frac{1}{16} e_{7}+\frac{\sqrt{3}}{16}+\frac{\sqrt{3}}{16} e_{3}\right) \\
=\frac{1}{16} e_{7}-\frac{\sqrt{3}}{16}+\frac{1}{16} e_{1}-\frac{\sqrt{3}}{16} e_{3}
\end{gathered}
$$

Lastly, we construct the octonions $V_{\nu \mu}$ for the muon neutrino and $V_{\nu \tau}$ for the tau neutrino, by left multiplying on the electron neutrino $V_{\nu}$ with $e^{2 \pi e_{6} / 3}$

$$
\begin{gathered}
\left(-\frac{1}{2}+\frac{\sqrt{3}}{2} e_{6}\right) \times \frac{1}{2} e_{6}=-\frac{1}{4} e_{6}-\frac{\sqrt{3}}{4} \\
V_{\nu \tau}=\left(-\frac{1}{2}+\frac{\sqrt{3}}{2} e_{6}\right) \times\left(-\frac{1}{4} e_{6}-\frac{\sqrt{3}}{4}\right)=-\frac{1}{4} e_{6}+\frac{\sqrt{3}}{4}
\end{gathered}
$$

We now have all the information needed to write down the four Jordan matrices whose eigenvalues we will calculate. Diagonal entries are electric charge, and off-diagonal entries are octonions representing the particles. Using the above results we write down these four matrices explicitly. The neutrinos of three generations

$$
X_{\nu}=\left[\begin{array}{ccc}
0 & V_{\nu} & V_{\nu \mu}^{*} \\
V_{\nu}^{*} & 0 & V_{\nu \tau} \\
V_{\nu \mu} & V_{\nu \tau}^{*} & 0
\end{array}\right]
$$

The anti-down set of quarks of three generations [anti-down, anti-strange, anti-bottom]:

$$
X_{a d}=\left[\begin{array}{ccc}
\frac{1}{3} & V_{a d} & V_{a s}^{*} \\
V_{a d}^{*} & \frac{1}{3} & V_{a b} \\
V_{a s} & V_{a b}^{*} & \frac{1}{3}
\end{array}\right]
$$


The up set of quarks for three generations [up, charm, top]

$$
X_{u}=\left[\begin{array}{ccc}
\frac{2}{3} & V_{u} & V_{c}^{*} \\
V_{u}^{*} & \frac{2}{3} & V_{t} \\
V_{c} & V_{t}^{*} & \frac{2}{3}
\end{array}\right]
$$

The positively charged leptons of three generations [positron, anti-muon, anti-tau-lepton]

$$
X_{e+}=\left[\begin{array}{ccc}
1 & V_{e+} & V_{a \mu}^{*} \\
V_{e+}^{*} & 1 & V_{a \tau} \\
V_{a \mu} & V_{\nu \tau}^{*} & 1
\end{array}\right]
$$

Next, the eigenvalue equation corresponding to each of these Jordan matrices can be written down, after using the expressions given above for calculating the determinant and the function $S(X)$. Tedious but straightforward calculations with the octonion algebra give the following four cubic equations:

Neutrinos: We get $\operatorname{Tr}(X)=0, S(X)=-3 / 4, \operatorname{Det}(X)=0$, and hence the cubic equation and roots

$$
\lambda^{3}-\frac{3}{4} \lambda=0 \quad \text { ROOTS }:\left(-2 \sqrt{\frac{3}{8}}, 0,2 \sqrt{\frac{3}{8}}\right)
$$

Anti-down-quark + its higher generations [anti-down, anti-strange, anti-bottom]: We get $\operatorname{Tr}(X)=1, S(X)=-1 / 24, \operatorname{Det}(X)=-19 / 216$, and the following cubic equation and roots

$$
\begin{array}{r}
\lambda^{3}-\lambda^{2}-\frac{1}{24} \lambda+\frac{19}{216}=0 \\
\text { ROOTS }: \frac{1}{3}-\sqrt{\frac{3}{8}}, \frac{1}{3}, \frac{1}{3}+\sqrt{\frac{3}{8}}
\end{array}
$$

Up quark + its higher generations [up, charm, top]: We get $\operatorname{Tr}(X)=2, S(X)=23 / 24, \operatorname{Det}(X)=$ 5/108 and the following cubic equation and roots:

$$
\begin{array}{r}
\lambda^{3}-2 \lambda^{2}+\frac{23}{24} \lambda-\frac{5}{108}=0 \\
\text { ROOTS }: \frac{2}{3}-\sqrt{\frac{3}{8}}, \frac{2}{3}, \frac{2}{3}+\sqrt{\frac{3}{8}}
\end{array}
$$

Positron + its higher generations [positron, anti-muon, anti-tau-lepton]: We get $\operatorname{Tr}(X)=$ 
$3, S(X)=3-3 / 32, \operatorname{Det}(X)=1-3 / 32$ and the following cubic equation and roots:

$$
\begin{array}{r}
\lambda^{3}-3 \lambda^{2}+\left(3-\frac{3}{32}\right) \lambda-\left(1-\frac{3}{32}\right)=0 \\
\text { ROOTS : } 1-\frac{1}{2} \sqrt{\frac{3}{8}}, 1,1+\frac{1}{2} \sqrt{\frac{3}{8}}
\end{array}
$$

As expected from the known elementary properties of cubic equations, the sum of the roots is $\operatorname{Tr}(X)$, their product is $\operatorname{Det}(X)$, and the sum of their pairwise products is $S(X)$. Interestingly, this also shows that the sum of the roots is equal to the total electric charge of the three fermions under consideration in each of the respective cases. Whereas $S(X)$ and $\operatorname{Det}(X)$ are respectively related to an invariant inner product and an invariant trilinear form constructed from the Jordan matrix, their physical interpretation in terms of fermion properties remains to be understood.

The roots exhibit a remarkable pattern. In each of the four cases, one of the three roots is equal to the corresponding electric charge, and the other two roots are placed symmetrically on both sides of the middle root, which is the one equal to the electric charge. All three roots are positive in the up quark set and in the positron set, whereas the neutrino set and anti-down quark set have one negative root each, and the neutrino also has a zero root. It is easily verified that the calculation of eigenvalues for the anti-particles yields the same set of eigenvalues., upto a sign.

One expects these roots to relate to masses of quarks and leptons for various reasons, and principally because the automorphism group of the complexified octonions contains the 4D Lorentz group as well, and the latter we know relates to gravity. Since mass is the source of gravity, we expect the Lorentz group to be involved in an essential way in any theory which predicts masses of elementary particles. And the group $F_{4}$, besides being related to $G_{2}$, and a possible candidate for the unification of the four interactions, is also the automorphism group of the EJA. We have motivated how the four projections of the EJA relate naturally to the four generation sets of the fermions. Thus there is a strong possibility that the eigenvalues of the characteristic equation of the EJA yield information about fermion mass ratios, especially it being a cubic equation with real roots. We make the following preliminary observations about the known mass ratios, in the hope that they might help give some further insight into the possible relevance of these eigenvalues. 
For the set (positron, anti-muon, anti-tau-lepton), the three respective masses are known to satisfy the following empirical relation, known as the Koide formula:

$$
\frac{m_{e}+m_{\mu}+m_{\tau}}{\left(\sqrt{m_{e}}+\sqrt{m_{\mu}}+\sqrt{m_{\tau}}\right)^{2}}=0.666661(7) \approx \frac{2}{3}
$$

For the three roots of the corresponding cubic equation (32) we get that

$$
2 \frac{\lambda_{1}^{2}+\lambda_{2}^{2}+\lambda_{3}^{2}}{\left(\lambda_{1}+\lambda_{2}+\lambda_{3}\right)^{2}}=2 \frac{[\operatorname{Tr}(X)]^{2}-2 S(X)}{[\operatorname{Tr}(X)]^{2}}=\frac{2}{3}\left(1+\frac{1}{16}\right) \approx 0.70833
$$

The factor $1 / 16$ comes from the sum of the absolute values of the three octonions which go into the related Jordan matrix. This observation suggests that the eigenvalues bear some relation with the square roots of the masses of the three charged leptons, though simply comparing square roots of their mass-ratios does not seem to yield any obvious relation with the eigenvalues. Further investigation is in progress. Rather, we get the following logarithmic ratios for masses of the charged leptons [taken as $0.5 \mathrm{MeV}, 105 \mathrm{MeV}, 1777 \mathrm{Mev}$ ] and for the roots

$$
\begin{gathered}
\ln \left(\frac{105}{0.5}\right)^{1 / 4} \sim 1.34 ; \quad \frac{1+\sqrt{\frac{3}{32}}}{1} \sim 1.31 \\
\ln \left(\frac{1777}{0.5}\right)^{1 / 4} \sim 2.04 ; \quad \frac{1+\sqrt{\frac{3}{32}}}{1-\sqrt{\frac{3}{32}}} \sim 1.88 \\
\ln \left(\frac{1777}{105}\right)^{1 / 4} \sim 0.70 ; \quad \frac{1+\sqrt{\frac{3}{32}}}{1-\sqrt{\frac{3}{32}}}-\frac{1+\sqrt{\frac{3}{32}}}{1} \sim 0.57
\end{gathered}
$$

For the up quark set though, we see a correlation in terms of square roots of masses.

In the case of the up quark set, the following approximate match is observed between the ratios of the eigenvalues, and the mass square root ratios of the masses of up, charm and top quark. For the sake of this estimate we take these three quark masses to be [2.3, 1275, 173210] in Mev [28]. The following ratios are observed:

$$
\sqrt{\frac{1275}{2.3}} \sim 23.55 ; \quad \frac{\frac{2}{3}+\sqrt{\frac{3}{8}}}{\frac{2}{3}-\sqrt{\frac{3}{8}}} \approx 23.56
$$




$$
\begin{gathered}
\sqrt{\frac{173210}{1275}} \sim 11.66 ; \quad \frac{\frac{2}{3}}{\frac{2}{3}-\sqrt{\frac{3}{8}}} \approx 12.28 \\
\sqrt{\frac{173210}{2.3}} \sim 274.42 ; \quad\left(\frac{\frac{2}{3}+\sqrt{\frac{3}{8}}}{\frac{2}{3}-\sqrt{\frac{3}{8}}}\right) \times\left(\frac{\frac{2}{3}}{\frac{2}{3}-\sqrt{\frac{3}{8}}}\right) \approx 289.23
\end{gathered}
$$

Within the error bars on the masses of the up set of quarks, the two sets of ratios are seen to agree with each other upto second decimal place.

Considering that one of the roots is negative in the anti-down-quark set, we have not succeeded in identifying any discernible correlation with mass ratios here. The same is true for the neutrino set, where one root is negative and one root is zero. Nonetheless, the case of the neutrino is instructive, and shows how non-zero mass could arise fundamentally, even when the electric charge is zero. In this case, the non-zero contribution comes from the inner product related quantity $S(X)$, and therein from the absolute magnitude of the octonions in the Jordan matrix, which necessarily has to be non-zero. We thus see that masses are derivative concepts, obtained from the three more fundamental entities, namely the electric charge, and the geometric invariants $S(X)$ and $\operatorname{Det}(X)$, with the last two necessarily being defined commonly for the three generations. And since mass is the source of gravity, this picture is consistent with gravity and space-time geometry being emergent from the underlying geometry of the octonionic space which algebraically determines the properties of the elementary particles. We note that there are no free parameters in the above analysis, no dimensional quantities, and no assumption has been put by hand. Except that we identify the octonions with elementary fermions. The numbers which come out from the above analysis are number-theoretic properties of the octonion algebra.

These observations suggest a possible fundamental relation between eigenvalues of the EJA and particle masses. In the next section, we provide preliminary, modest, evidence for such a connection, based on our proposal for unification based on division algebras and a matrix-valued Lagrangian dynamics. 


\section{AN OCTONIONIC LAGRANGIAN FOR THE STANDARD MODEL}

The action and Lagrangian for the three generations of standard model fermions, fourteen gauge bosons, and four potential Higgs bosons, are given by [24]

$$
\frac{S}{C_{0}}=\int d \tau \mathcal{L} \quad ; \quad \mathcal{L}=\frac{1}{2} \operatorname{Tr}\left[\frac{L_{p}^{2}}{L^{2}} \dot{\widetilde{Q}}_{1}^{\dagger} \dot{\widetilde{Q}}_{2}\right]
$$

Here,

$$
\dot{\widetilde{Q}}_{1}^{\dagger}=\dot{\widetilde{Q}}_{B}^{\dagger}+\frac{L_{p}^{2}}{L^{2}} \beta_{1} \dot{\widetilde{Q}}_{F}^{\dagger} ; \quad \dot{\widetilde{Q}}_{2}=\dot{\widetilde{Q}}_{B}+\frac{L_{p}^{2}}{L^{2}} \beta_{2} \dot{\widetilde{Q}}_{F}
$$

and

$$
\dot{\widetilde{Q}}_{B}=\frac{1}{L}\left(i \alpha q_{B}+L \dot{q}_{B}\right) ; \quad \dot{\widetilde{Q}}_{F}=\frac{1}{L}\left(i \alpha q_{F}+L \dot{q}_{F}\right)=
$$

By defining

$$
q_{1}^{\dagger}=q_{B}^{\dagger}+\frac{L_{P}^{2}}{L^{2}} \beta_{1} q_{F}^{\dagger} \quad ; \quad q_{2}=q_{B}+\frac{L_{P}^{2}}{L^{2}} \beta_{2} q_{F}
$$

we can express the Lagrangian as

$$
\begin{aligned}
\mathcal{L} & =\frac{L_{P}^{2}}{2 L^{2}} \operatorname{Tr}\left[\left(\dot{q}_{1}^{\dagger}+\frac{i \alpha}{L} q_{1}^{\dagger}\right) \times\left(\dot{q}_{2}+\frac{i \alpha}{L} q_{2}\right)\right] \\
& =\frac{L_{P}^{2}}{2 L^{2}} \operatorname{Tr}\left[\dot{q}_{1}^{\dagger} \dot{q}_{2}-\frac{\alpha^{2}}{L^{2}} q_{1}^{\dagger} q_{2}+\frac{i \alpha}{L} q_{1}^{\dagger} \dot{q}_{2}+\frac{i \alpha}{L} \dot{q}_{1}^{\dagger} q_{2}\right]
\end{aligned}
$$

We now expand each of these four terms inside of the trace Lagrangian, using the definitions of $q_{1}$ and $q_{2}$ given above:

$$
\begin{aligned}
& \dot{q}_{1}^{\dagger} \dot{q}_{2}=\dot{q}_{B}^{\dagger} \dot{q}_{B}+\frac{L_{P}^{2}}{L^{2}} \dot{q}_{B}^{\dagger} \beta_{2} \dot{q}_{F}+\frac{L_{P}^{2}}{L^{2}} \beta_{1} \dot{q}_{F}^{\dagger} \dot{q}_{B}+\frac{L_{P}^{4}}{L^{4}} \beta_{1} \dot{q}_{F}^{\dagger} \beta_{2} \dot{q}_{F} \\
& q_{1}^{\dagger} q_{2}=q_{B}^{\dagger} q_{B}+\frac{L_{P}^{2}}{L^{2}} q_{B}^{\dagger} \beta_{2} q_{F}+\frac{L_{P}^{2}}{L^{2}} \beta_{1} q_{F}^{\dagger} q_{B}+\frac{L_{P}^{4}}{L^{4}} \beta_{1} q_{F}^{\dagger} \beta_{2} q_{F} \\
& q_{1}^{\dagger} \dot{q}_{2}=q_{B}^{\dagger} \dot{q}_{B}+\frac{L_{P}^{2}}{L^{2}} q_{B}^{\dagger} \beta_{2} \dot{q}_{F}+\frac{L_{P}^{2}}{L^{2}} \beta_{1} q_{F}^{\dagger} \dot{q}_{B}+\frac{L_{P}^{4}}{L^{4}} \beta_{1} q_{F}^{\dagger} \beta_{2} \dot{q}_{F} \\
& \dot{q}_{1}^{\dagger} q_{2}=\dot{q}_{B}^{\dagger} q_{B}+\frac{L_{P}^{2}}{L^{2}} \dot{q}_{B}^{\dagger} \beta_{2} q_{F}+\frac{L_{P}^{2}}{L^{2}} \beta_{1} \dot{q}_{F}^{\dagger} q_{B}+\frac{L_{P}^{4}}{L^{4}} \beta_{1} \dot{q}_{F}^{\dagger} \beta_{2} q_{F}
\end{aligned}
$$

In our recent work, we suggested this Lagrangian, having the symmetry group $F_{4}$, as a candidate for unification. There are fourteen gauge bosons (equal to the number of generators of $G_{2}$ ). These are the eight gluons, the three weak isospin vector bosons, the photon, and the two Lorentz bosons. These bosons, along with one Higgs, can be accounted for by the four 
bosonic terms which form the first column in the above four sub-equations. The remaining twelve terms were proposed to describe three fermion generations and three Higgs, with the three generations being motivated by the triality of $S O(8)$. However, one important question which has not been addressed there is: why does triality not give rise to three copies of the bosons?! In the framework of the present approach we tentatively explore the following answer. We know that the even-grade Grassmann numbers which form the entries of the bosonic matrices are made from even-number products of odd-grade (fermionic) Grassmann numbers, and the latter are in a sense more basic. Could it then be that bosonic degrees of freedom are made from fermionic degrees of freedom? If this were to be so, it could prevent the tripling of bosons, if we think of them as arising at the 'intersections' of the octonionic directions which represent fermions.

The seven imaginary unit octonions are used to make the Fano plane, which has seven points and seven lines [adding to fourteen elements; points and lines have equal status]. If we include the real direction [we have assumed $\dot{q}_{B 0}$ to be self-adjoint] also, we get an equivalent of a 3-D cube where the eight vertices now stand for the eight octonions, with one of them [the 'origin'] standing for the real line. As explained by Baez: "The Fano plane is the projective plane over the 2-element field $Z_{2}$. In other words, it consists of lines through the origin in the vector space $Z_{2}^{3}$. Since every such line contains a single nonzero element, we can also think of the Fano plane as consisting of the seven nonzero elements of $Z_{2}^{3}$. If we think of the origin in $Z_{2}^{3}$ as corresponding to 1 in $\mathbb{O}$, we get the following picture of the octonions". This picture is Fig. 3 below, borrowed from Baez [14]. Considering points, lines and faces together, this structure has 26 elements $[8+12+6=26]$. Motivated by this representation of the octonion, and the triality of $S O(8)$, we propose the following diagrammatic representation of the standard model fermions, gauge bosons, and Higgs as shown in Fig. 4. It motivates us to think of bosons as arising as 'intersections' of the elements representing fermions. We have taken four copies of the Baez cube, with the central one at the intersection of the other three, and used them to represent the elementary particles. We now attempt to describe Fig. 4 in some detail. There is a central black-colored cube (henceforth a cube is an octonion) in the front, which represents the fourteen gauge bosons and the four Higgs bosons; we will return to this cube shortly. Then there are three more (colored) cubes: one to the left, one at the back, and one at the bottom. These are marked as Gen I, Gen II and Gen III, and represent the three fermion generations. Let us focus first on the octonion on the left, 


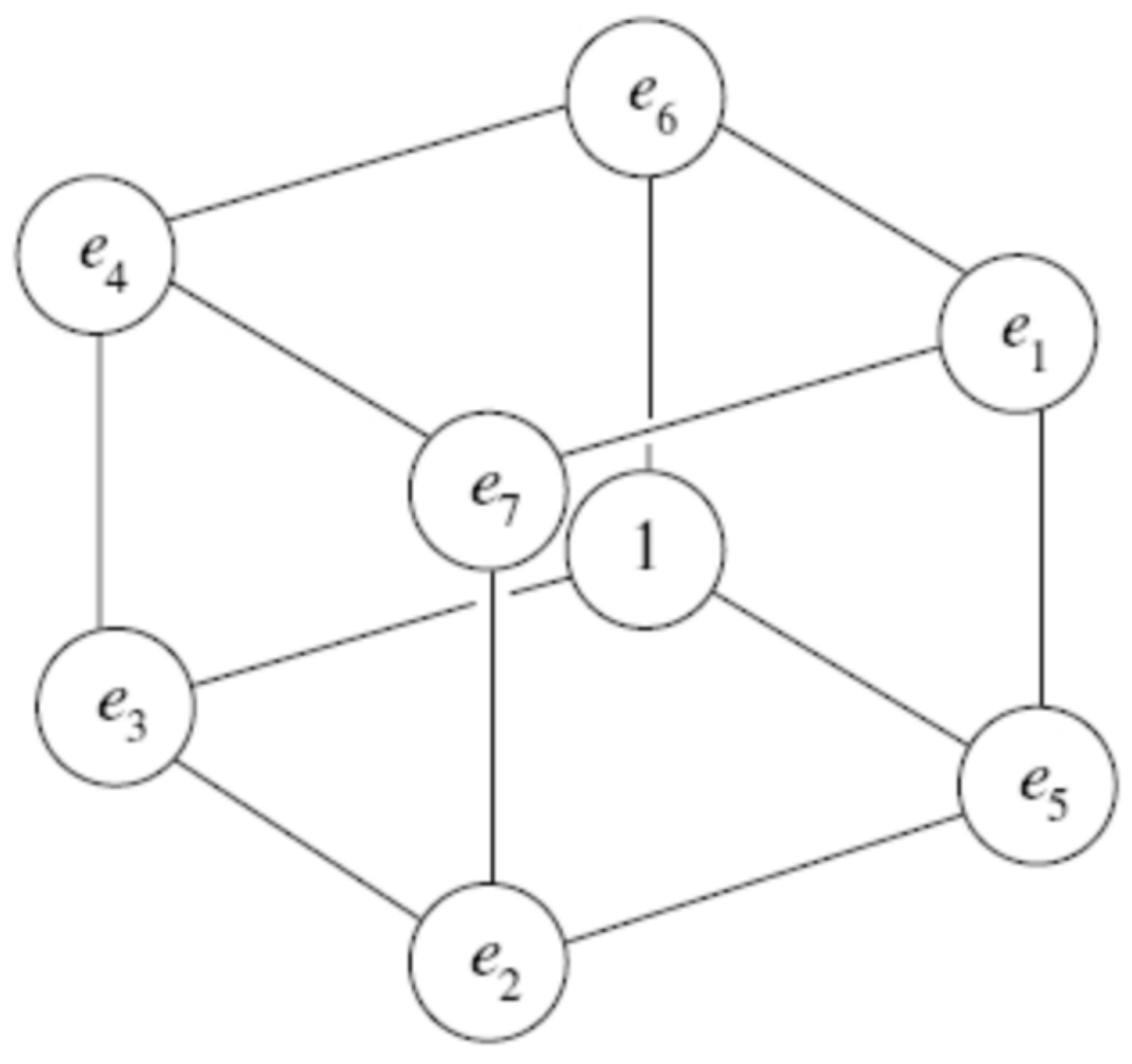

FIG. 3. The octonions [From Baez [14]].

which is Gen I, and where the eight vertices have been marked $\left(e_{0}, e_{1}, e_{2}, e_{3}, e_{4}, e_{5}, e_{6}, e_{7}\right)$ just as in the Baez cube. If $e_{0}$ were to be excluded, this cube becomes the Fano plane [Fig. 1 above] and the arrows marked in the Gen. I cube follow the same directions as in the Fano plane. In this Gen I cube, leaving out all those elements which are at the intersection with the central bosonic cube, and leaving out the face on the far left, we are left with sixteen elements: four points, eight lines, and four faces. The four points are shown in blue and are $\left(e_{3}, e_{5}, e_{6}, e_{7}\right)$. The eight lines are: $\left(e_{4} e_{3}, e_{7} e_{2}, e_{3} e_{7}, e_{7} e_{6}, e_{5} e_{6}, e_{6} e_{4}, e_{5} e_{0}, e_{6} e_{1}\right)$. The four planes are: $\left(e_{4} e_{3} e_{7} e_{2}\right),\left(e_{0} e_{5} e_{6} e_{1}\right),\left(e_{7} e_{2} e_{1} e_{6}\right),\left(e_{3} e_{4} e_{0} e_{5}\right)$. Between them, these sixteen elements represent the eight fermions and their anti-particles in one generation, one particle / anti-particle per octonionic element.

The up quark, the down quark, and their anti-particles of one particular color are (marked 


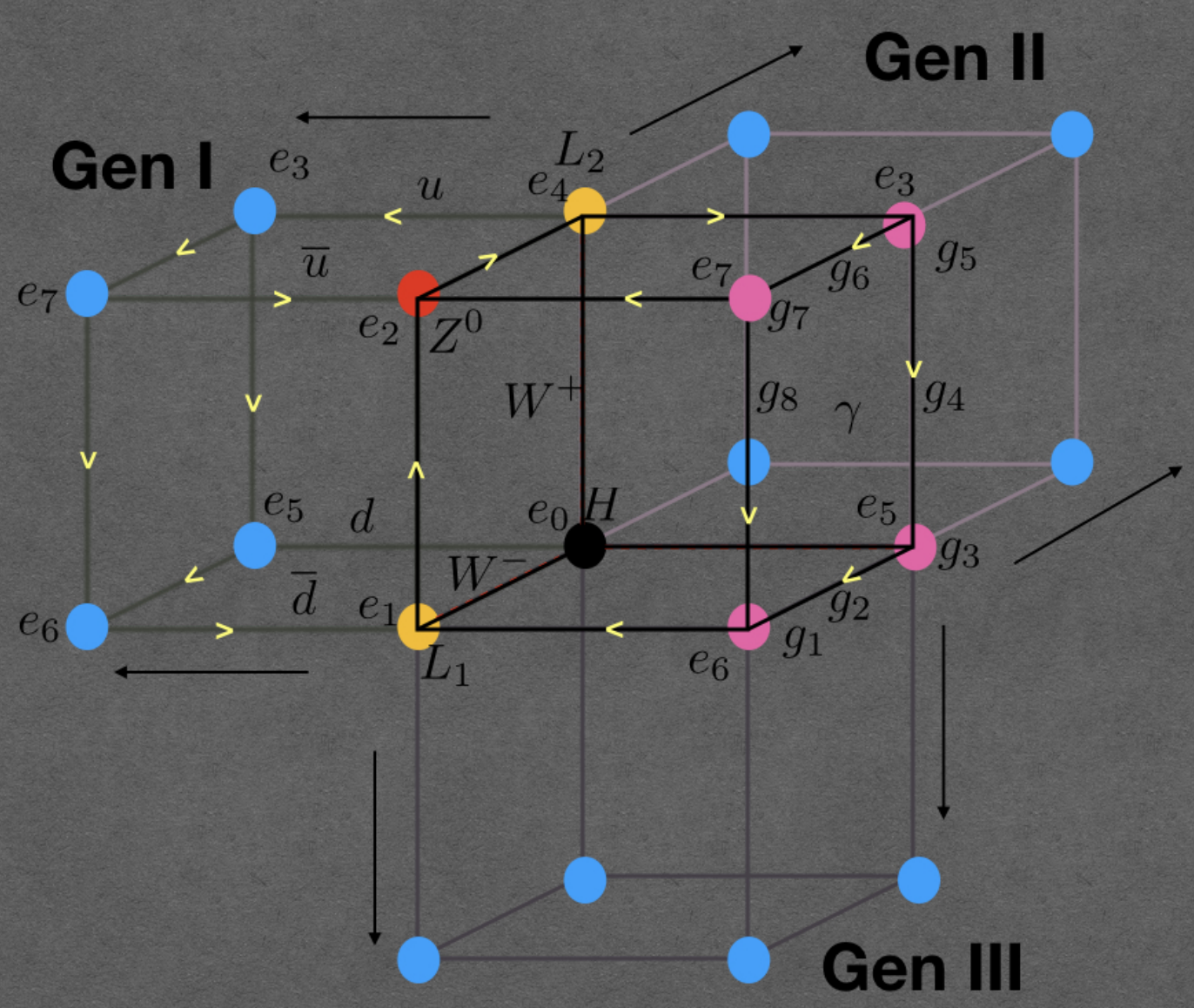

FIG. 4. The elementary particles of the standard model with three generations, represented through octonions in an $F_{4}$ diagram.. Please see text for a detailed explanation.

by) the four lines $\left(e_{4} e_{3}, e_{7} e_{2}, e_{0} e_{5}, e_{6} e_{1}\right)$. The points $\left(e_{3}, e_{5}, e_{6}, e_{7}\right)$ mark $u, d$ of a second color, and the lines $\left(e_{3} e_{7}, e_{7} e_{6}, e_{3} e_{5}, e_{5} e_{6}\right)$ mark the $u, d$ of the third color. The four planes mark the electron, the neutrino, and their anti-particles. Between them, these sixteen elements have an $S U(3)$ symmetry: they can be correlated to the $(8+8) \mathrm{D}$ particle basis constructed by Furey, from the $S U(3)$ in $G_{2}$. Next, the Gen II and Gen III along with Gen I has another $S U(3)$ symmetry, which is responsible for the three generations. These three fermionic cubes represent three intersecting copies of $G_{2}$ each cube having an $S U(3)$ symmetry. The three-way intersection is $S U(2) X S U(2)$, this being the black central cube, and the bosons lie on this cube. At the same time the fermionic cubes make contact with the bosonic cube, enabling the bosons to act on the fermions.

We now try to understand the central bosonic cube. First we count the number of its elements: it gets a total of $3 \times 10=30$ elements from the three side cubes, which when added 
to its own 26 elements gives a total of 56. But there are a lot of common elements, so that the actual number of independent elements is much smaller, and we enumerate them now. Three points are shared two-way and three points shared three-way and the point $e_{0}$ is shared four-way; that reduces the count to 44. Nine lines are shared: three of them three way, and six of them two way, reducing the count to 32 . The shared three planes reduce the count to 29. We now account for the assignment of bosons to these 29 locations.

The eight gluons are on the front right, marked by the pink points, and lines labelled $g_{1}$ to $g_{8}$, and the photon is assigned to the plane $\left(e_{3} e_{7} e_{6} e_{5}\right)$ on the front right enclosed by the gluons. The two Lorentz bosons are the yellow points $e_{4}$ and $e_{1}$ also marked $L_{2}$ and $L_{1}$. The three vector bosons are marked by the lines $e_{0} e_{1}, e_{0} e_{4}$ and the point $e_{2}$, also marked $Z^{0}$. The Higgs $H$ is at the four way real point $e_{0}$. Three more Higgs are shown as follows: two planes per Higgs, e.g. the plane $e_{0} e_{4} e_{2} e_{1}$ and the mirror fermionic plane $e_{3} e_{5} e_{6} e_{7}$ on the far left in Gen I. Analogously, another Higgs is given by the bosonic plane $e_{0} e_{1} e_{6} e_{5}$ and its mirror fermionic plane at the front bottom in Gen III. The third Higgs is given by the bosonic plane $e_{0} e_{4} e_{3} e_{5}$ and its mirror fermionic plane at the back in Gen II. This way 21 elements are used up. The remaining 8 un-used elements (six lines and two planes) are assigned to eight terms in the Lagrangian representing the action of the spacetime symmetry on the gluons: these are the terms $\dot{q}_{B} q_{B}^{\dagger}$ and $\dot{q}_{B}^{\dagger} q_{B}$ in (48).

The bosonic cube lies in the intersection of the three $G_{2}$ and hence does not triplicate during the $S U(3)$ rotation which generates the three fermion generations. The symmetry group of the theory is the 52 dimensional group $F_{4}$, with $8 \times 3=24$ generators coming from the three fermionic cubes, and the rest 28 from the bosonic sector $[14+2 \mathrm{x} 3+8=28]$. This diagram does suggest that one could investigate bosonic degrees of freedom as made from pairs of fermion degrees of freedom. With this tentative motivation, we return to our Lagrangian, and seek to write it explicitly as for a single generation of bosons, and three generations of fermions. Upon examination of the sub-equations in Eqn. (48) we find that the last column has terms bilinear in the fermions, and we would like to make it appear just as the second and third column do, so that we can explicitly have three fermion generations. With this intent, we propose the following assumed definitions of the bosonic degrees of 
freedom, by recasting the four terms in the last column of Eqn. (48):

$$
\begin{aligned}
& \frac{L_{P}^{4}}{L^{4}} \beta_{1} \dot{q}_{F}^{\dagger} \beta_{2} \dot{q}_{F} \equiv \frac{L_{P}^{2}}{L^{2}} \dot{q}_{B} \beta_{2} \dot{q}_{F}+\frac{\alpha^{2}}{L^{2}} A \\
& \frac{L_{P}^{4}}{L^{4}} \beta_{1} q_{F}^{\dagger} \beta_{2} q_{F} \equiv \frac{L_{P}^{2}}{L^{2}} q_{B} \beta_{2} q_{F}+A \\
& \frac{L_{P}^{4}}{L^{4}} \beta_{1} q_{F}^{\dagger} \beta_{2} \dot{q}_{F} \equiv \frac{L_{P}^{2}}{L^{2}} q_{B}^{\dagger} \beta_{1} \dot{q}_{F}^{\dagger}+B \\
& \frac{L_{P}^{4}}{L^{4}} \beta_{1} \dot{q}_{F}^{\dagger} \beta_{2} q_{F} \equiv \frac{L_{P}^{2}}{L^{2}} \dot{q}_{B}^{\dagger} \beta_{1} q_{F}^{\dagger}-B
\end{aligned}
$$

where $A$ and $B$ are bosonic matrices which drop out on summing the various terms to get the full Lagrangian, With this redefinition, the sub-equations Eqn. (48) can be now written in the following form after rewriting the last column:

$$
\begin{aligned}
& \dot{q}_{1}^{\dagger} \dot{q}_{2}=\dot{q}_{B}^{\dagger} \dot{q}_{B}+\frac{L_{P}^{2}}{L^{2}} \dot{q}_{B}^{\dagger} \beta_{2} \dot{q}_{F}+\frac{L_{P}^{2}}{L^{2}} \beta_{1} \dot{q}_{F}^{\dagger} \dot{q}_{B}+\frac{L_{P}^{2}}{L^{2}} \dot{q}_{B} \beta_{2} \dot{q}_{F} \\
& q_{1}^{\dagger} q_{2}=q_{B}^{\dagger} q_{B}+\frac{L_{P}^{2}}{L^{2}} q_{B}^{\dagger} \beta_{2} q_{F}+\frac{L_{P}^{2}}{L^{2}} \beta_{1} q_{F}^{\dagger} q_{B}+\frac{L_{P}^{2}}{L^{2}} q_{B} \beta_{2} q_{F} \\
& q_{1}^{\dagger} \dot{q}_{2}=q_{B}^{\dagger} \dot{q}_{B}+\frac{L_{P}^{2}}{L^{2}} q_{B}^{\dagger} \beta_{2} \dot{q}_{F}+\frac{L_{P}^{2}}{L^{2}} \beta_{1} q_{F}^{\dagger} \dot{q}_{B}+\frac{L_{P}^{2}}{L^{2}} q_{B}^{\dagger} \beta_{1} \dot{q}_{F}^{\dagger} \\
& \dot{q}_{1}^{\dagger} q_{2}=\dot{q}_{B}^{\dagger} q_{B}+\frac{L_{P}^{2}}{L^{2}} \dot{q}_{B}^{\dagger} \beta_{2} q_{F}+\frac{L_{P}^{2}}{L^{2}} \beta_{1} \dot{q}_{F}^{\dagger} q_{B}+\frac{L_{P}^{2}}{L^{2}} \dot{q}_{B}^{\dagger} \beta_{1} q_{F}^{\dagger}
\end{aligned}
$$

The terms now look harmonious and we can see a structure emerging - the first column are bosonic terms and these are not triples. The remaining terms are four sets of three each [to which their adjoints will eventually get added] which can clearly describe three generations of the four sets, which is what we had in the Jordan matrices in the previous section. Putting it all together, we can now rewrite the Lagrangian so that it explicitly looks like the one for gauge bosons and four sets of three generations of fermions, as in the Jordan matrix:

$$
\begin{gathered}
\mathcal{L}=\frac{L_{P}^{2}}{2 L^{2}} \operatorname{Tr}\left[\left(\dot{q}_{1}^{\dagger}+\frac{i \alpha}{L} q_{1}^{\dagger}\right) \times\left(\dot{q}_{2}+\frac{i \alpha}{L} q_{2}\right)\right] \\
=\frac{L_{P}^{2}}{2 L^{2}} \operatorname{Tr}\left[\dot{q}_{1}^{\dagger} \dot{q}_{2}-\frac{\alpha^{2}}{L^{2}} q_{1}^{\dagger} q_{2}+\frac{i \alpha}{L} q_{1}^{\dagger} \dot{q}_{2}+\frac{i \alpha}{L} \dot{q}_{1}^{\dagger} q_{2}\right] \\
\equiv \frac{L_{P}^{2}}{2 L^{2}} \operatorname{Tr}\left[\mathcal{L}_{\text {bosons }}+\mathcal{L}_{\text {set } 1}+\mathcal{L}_{\text {set } 2}+\mathcal{L}_{\text {set } 3}+\mathcal{L}_{\text {set } 4}\right]
\end{gathered}
$$

where

$$
\mathcal{L}_{\text {bosons }}=\dot{q}_{B}^{\dagger} \dot{q}_{B}-\frac{\alpha^{2}}{L^{2}} q_{B}^{\dagger} q_{B}+\frac{i \alpha}{L} q_{B}^{\dagger} \dot{q}_{B}+\frac{i \alpha}{L} \dot{q}_{B}^{\dagger} q_{B}
$$




$$
\begin{gathered}
\mathcal{L}_{\text {set } 1}=\frac{L_{P}^{2}}{L^{2}} \dot{q}_{B}^{\dagger} \beta_{2} \dot{q}_{F}+\frac{L_{P}^{2}}{L^{2}} \beta_{1} \dot{q}_{F}^{\dagger} \dot{q}_{B}+\frac{L_{P}^{2}}{L^{2}} \dot{q}_{B} \beta_{2} \dot{q}_{F} \\
\mathcal{L}_{\text {set } 2}=-\frac{\alpha^{2}}{L^{2}}\left(\frac{L_{P}^{2}}{L^{2}} q_{B}^{\dagger} \beta_{2} q_{F}+\frac{L_{P}^{2}}{L^{2}} \beta_{1} q_{F}^{\dagger} q_{B}+\frac{L_{P}^{2}}{L^{2}} q_{B} \beta_{2} q_{F}\right) \\
\mathcal{L}_{\text {set } 3}=\frac{i \alpha}{L}\left(\frac{L_{P}^{2}}{L^{2}} q_{B}^{\dagger} \beta_{2} \dot{q}_{F}+\frac{L_{P}^{2}}{L^{2}} \beta_{1} q_{F}^{\dagger} \dot{q}_{B}+\frac{L_{P}^{2}}{L^{2}} q_{B}^{\dagger} \beta_{1} \dot{q}_{F}^{\dagger}\right) \\
\mathcal{L}_{\text {set } 4}=\frac{i \alpha}{L}\left(\frac{L_{P}^{2}}{L^{2}} \dot{q}_{B}^{\dagger} \beta_{2} q_{F}+\frac{L_{P}^{2}}{L^{2}} \beta_{1} \dot{q}_{F}^{\dagger} q_{B}+\frac{L_{P}^{2}}{L^{2}} \dot{q}_{B}^{\dagger} \beta_{1} q_{F}^{\dagger}\right)
\end{gathered}
$$

We see that each of these four fermionic sets could possibly be related to a Jordan matrix, after including the adjoint part. We also see that different coupling constants appear in different sets with identical coupling in third and fourth set and no coupling in the first set. The first set could possibly describe neutrinos (only gravitational and weak interaction), the second set charged leptons, and the third and fourth set the quarks. To establish this, equations of motion remain to be worked out and then related to the eigenvalue problem. As noted earlier, $L$ relates to mass, and this approach could reveal how the eigenvalues of the EJA characteristic equation relate to mass. This investigation is currently in progress.

The Jordan eigenvalues and the low energy limiting value of the fine structure constant : If we examine the Lagrangian term for the charged leptons in Eqn. (52), the dimensionless coupiing constant $C$ in front of it is (upto a sign):

$$
C \equiv \alpha^{2} \frac{L_{P}^{4}}{L^{4}}
$$

[The operator terms of the form $q_{B} q_{F}$ etc. in (52) have been correspondingly made dimensionless by dividing by $\left.L_{P}^{2}\right]$. We assume that $\ln \alpha$ is linearly proportional to the electric charge, and that the proportionality constant is the Jordan eigenvalue corresponding to the anti-down quark. The electric charge 1/3 of the anti-down quark seems to be the right choice for determining $\alpha$, it being the smallest non-zero value [and hence possibly the fundamental value] of the electric charge, and also because the constant $\alpha$ appears as the coupling in front of the supposed quark terms in the Lagrangian, as in Eqns. (53) and (54). We hence define $\alpha$ by

$$
\ln \alpha \equiv \lambda_{a d} q_{a d}=\left[\frac{1}{3}-\sqrt{\frac{3}{8}}\right] \times \frac{1}{3} \quad \Longrightarrow \quad \alpha^{2} \approx=0.83025195149
$$


where $\lambda_{a d}$ is the Jordan eigenvalue corresponding to the anti-down quark, as given by Eqn. (30) and $q_{a d}$ is the electric charge of the anti-down quark $(=1 / 3)$.

As for the value of $L_{P} / L$, we identify it with that part of the Jordan eigenvalue which modifies the contribution coming from the electric charge. Thus from the eigenvalues found above, we deduce that for neutrinos, quarks and charged leptons, the quantity $L_{P}^{2} / L^{2}$ takes the respective values $(3 / 2,3 / 8,3 / 32)$. Thus the coupling constant $C$ defined above can now be calculated, with $\alpha^{2}$ as given above, and $L_{P}^{2} / L^{2}=3 / 32$. Furthermore, since the electric charge $q$, the way it is conventionally defined, has dimensions such that $q^{2}$ has dimensions $\left(\right.$ Energy $\times$ Length), we measure $q^{2}$ in Planck units $E_{P l} \times L_{P}=\hbar c$. We hence define the fine structure constant by $C=\alpha^{2} L_{P}^{4} / L^{4} \equiv e^{2} / \hbar c$, where $e$ is the electric charge of electron / muon / tau-lepton in conventional units. We hence get the value of the fine structure constant to be

$$
C=\alpha^{2} L_{P}^{4} / L^{4} \equiv e^{2} / \hbar c=\exp \left[\left[\frac{1}{3}-\sqrt{\frac{3}{8}}\right] \times \frac{2}{3}\right] \times \frac{9}{1024} \approx 0.00729713=\frac{1}{137.04006}
$$

The CODATA 2018 value of the fine structure constant is

$$
0.0072973525693(11)=1 / 137.035999084(21)
$$

Our calculated value differs from the measured value in the seventh decimal place. In the next section, we show how incorporating the Karolyhazy length correction gives an exact match with the CODATA 2018 value, if we assume a specific value for the electro-weak symmetry breaking energy scale.

With this value of $\alpha$, the magnitude of the corresponding dimensionless coupling for the supposed quark terms (53) and (54) is given by, with $L_{P}^{2} / L^{2}=3 / 8$,

$$
\alpha^{2} \frac{L_{P}^{4}}{L^{4}}=0.8302 \times\left(\frac{3}{8}\right)^{2} \approx 0.1167
$$

This compares well with the measured QCD coupling constant at about $90 \mathrm{GeV}$. The possible relevance of this result to the runnnig coupling of QCD remains to be understood. We note that the relative strength of the electromgnetic coupling and the QCD coupling [for these limiting values] is $(9 / 1024) /(9 / 64)=1 / 16$. 
Once a theoretical derivation of the asymptotic fine structure constant is known, one can write the electric charge $e$ as

$$
e=(3 / 32) \exp [1 / 9-1 / \sqrt{24}]\left(\hbar L_{P} / t_{P}\right)^{1 / 2}
$$

where $L_{P}$ and $t_{P}$ are Planck length and Planck time respectively - obviously their ratio is the speed of light. In our theory, there are only three fundamental dimensionful quantities: Planck length, Planck time, and a constant with dimensions of action, which in the emergent quantum theory is identified with Planck's constant $\hbar$. We now see that electric charge is not independent of these three fundamental dimensionful constants. It follows from them. Planck mass is also constructed from these three, and electron mass will be expressed in terms of Planck mass, if only we could understand why the electron is some $10^{22}$ times lighter than Planck mass. Such a small number cannot come from the octonion algebra. In all likelihood, the cosmological expansion up until the electroweak symmetry breaking is playing a role here.

Thus electric charge and mass can both be expressed in terms of Planck's constant, Planck length and Planck time. This encourages us to think of electromagnetism, as well the other internal symmetries, entirely in geometric terms. This geometry is dictated by the $F_{4}$ symmetry of the exceptional Jordan algebra.

\section{CONCLUDING REMARKS}

We have not addressed the question as to how these discrete order one eigenvalues might relate to actual low values of fermion masses, which are much lower than Planck mass. We speculatively suggest the following scenario, which needs to be explored further. The universe is eight-dimensional, not four. The other four internal dimensions are not compactified; rather the universe is very 'thin' in those dimensions but they are expanding as well. There are reasons having to do with the so-called Karolyhazy uncertainty relation [29], because of which the universe expands in the internal dimensions at one-third the rate, on the logarithmic scale, compared to our $3 \mathrm{D}$ space. That is, if the $4 \mathrm{D}$ scale factor is $a(\tau)$, the internal scale factor is $a_{i n t}^{1 / 3}(\tau)$, in Planck length units. Taking the size of the observed universe to be about $10^{61}$ Planck units, the internal dimensions have a width approximately 
$10^{20}$ Planck units, which is about $10^{-13} \mathrm{~cm}$, thus being in the quantum domain. Classical systems have an internal dimension width much smaller than Planck length, and hence they effectively stay in [and appear to live in] four dimensional space-time. Quantum systems probe all eight dimensions, and hence live in an octonionic universe.

The universe began in a unified phase, via an inflationary $8 \mathrm{D}$ expansion possibly resulting as the aftermath of a huge spontaneous localisation event in a 'sea of atoms of space-timematter' [30]. The mass values are set, presumably in Planck scale, at order one values dictated by the eigenvalues reported in the present paper. Cosmic inflation scales down these mass values at the rate $a^{1 / 3}(\tau)$, where $a(\tau)$ is the $4 \mathrm{D}$ expansion rate. Inflation ends after about sixty e-folds, because seeding of classical structures breaks the color-elctro-weakLorentz symmetry, and classical spacetime emerges as a broken Lorentz symmetry. The electro-weak symmetry breaking is actually a electro-weakLorentz symmetry breaking, which is responsible for the emergence of gravity, weak interaction being its short distance limit. There is no reheating after inflation; rather inflation resets the Planck scale in the vicinity of the electro-weak scale, and the observed low fermion mass values result. The electroweak symmetry breaking is mediated by the Lorentz symmetry, in a manner consistent with the conventional Higgs mechanism. It is not clear why inflation should end specifically at the electro-weak scale: this is likely dictated by when spontaneous localisation becomes significant enough for classical spacetime to emerge. It is a competition between the strength of the electro-colour interaction which attempts to bind the fermions, and the inflationary expansion which opposes this binding. Eventually, the expanding universe cools enough for spontaneous localisation to win, so that the Lorentz symmetry is broken. It remains to prove from first principles that this happens at around the electro-weak scale and also to investigate the possibly important role that Planck mass primordial black holes might play in the emergence of classical spacetime. I would like to thank Roberto Onofrio for correspondence which has influenced these ideas. See also [31].

The Karolyhazy correction to the asymptotic value of fine structure constant: In accordance with the Karolyhazy uncertainty relation (Eqn. (9) of [29]) a measured length $l$ has a 'quantum gravitational' correction $\Delta l$ given by

$$
(\Delta l)^{3}=L_{P}^{2} l
$$


For the purpose of the present discussion we shall assume an equality sign here, i.e. that the numerical constant of proportionality between the two sides of the equation is unity. And, for the sake of the present application to the fine structure constant, we rewrite this relation as

$$
\delta \equiv \frac{L_{P}}{\Delta l}=\left(\frac{L_{P}}{l}\right)^{1 / 3}
$$

We set $l \equiv l_{f}$ where $l_{f}$ is the length scale $\left(\approx 10^{-16} \mathrm{~cm}\right)$ associated with electro-weak symmetry breaking, where classical space-time emerges from the prespacetime, prequantum theory. The assumption being that when the universe evolves from the Planck scale to the electro-weak scale [while remaining in the unbroken symmetry phase], the inverse of the octonionic length associated with the charged leptons (this being $\sqrt{3 / 32}$ ) is reset, because of the Karolyhazy correction, to

$$
\sqrt{\frac{3}{32}} \longrightarrow \sqrt{\frac{3}{32}}+\delta_{f} \equiv \sqrt{\frac{3}{32}}+\left(\frac{L_{P}}{l_{f}}\right)^{1 / 3}
$$

We can also infer this corrected length as the four-dimensional space-time measure of the length, which differs from the eight dimensional octonionic value $\sqrt{3 / 32}$ by the amount $\delta_{f}$. If we take $l_{f}$ to be $10^{-16} \mathrm{~cm}$, the correction $\delta_{f}$ is of the order $2 \times 10^{-6}$. The correction to the asymptotic value (57) of the fine structure constant is then

$$
C=\alpha^{2} L_{P}^{4} / L^{4} \equiv e^{2} / \hbar c=\alpha^{2}\left[\sqrt{\frac{3}{32}}+\left(\frac{L_{P}}{l_{f}}\right)^{1 / 3}\right]^{4}
$$

For $l_{f}=10^{-16} \mathrm{~cm}=198 \mathrm{GeV}^{-1}$, we get the corrected value of the fine structure constant to be 0.00729737649 , which overshoots the measured CODATA 2018 value at the eighth decimal place. The electroweak scale is generally assumed to lie in the range $100-1000 \mathrm{GeV}$. The value $l_{f}=1.3699526 \times 10^{-16} \mathrm{~cm}=144.530543605 \mathrm{GeV}^{-1}$ reproduces the CODATA 2018 value 0.0072973525693 of the asymptotic fine structure constant. The choice $l_{f}^{-1}=246 \mathrm{GeV}$ gives the value 0.00729739452 , whereas the choice $l_{f}^{-1}=159.5 \pm 1.5 \mathrm{GeV}$ gives the range (0.00729736049, 0.00729735908). $100 \mathrm{GeV}$ gives the value 0.00729732757 which is smaller than the measured value. $1000 \mathrm{GeV}$ gives 0.00729754842 . Thus in the entire $100-1000$ $\mathrm{GeV}$ range, the derived constant agrees with the measured value at least to the sixth decimal place, which is reassuring. The purpose of the present exercise is to show that the Karolyhazy 
correction leads to a correction to the asymptotic value of the fine structure constant which is in the desired range - a striking fact by itself. In principle, our theory should predict the precise value of the electroweak symmetry breaking scale. Since that analysis has not yet been carried out, we predict that the ColorElectro-WeakLorentz symmetry breaking scale is 144.something $\mathrm{GeV}$, because only then the theoretically calculated value of the asymptotic fine structure constant matches the experimentally measured value.

The above discussion of the asymptotic low energy value of the fine structure constant should not be confused with the running of the constant with energy. Once we recover classical spacetime and quantum field theory from our theory, after the ColorElectro-WeakLorentz symmetry breaking, conventional RG arguments apply, and the running of couplings with energy is to be worked out as is done conventionally. Such an analysis of running couplings will however be valid only up until the broken symmnetry is restored - it is not applicable in the prespacetime prequantum phase. In this sense, our theory is different from GUTs. Once there is unification, Lorentz symmetry is unified with internal symmetries - the exact energy scale at which that happens remains to be worked out.

How then does the Planck scale prespacetime, prequantum theory know about the low energy asymptotic value of the fine structure constant? The answer to this question lies in the Lagrangian given in (49) and in particular the Lagrangian term (52) for the charged leptons. In determining the asymptotic fine structure constant from here, we have neglected the modification to the coupling that will come from the presence of $q_{B}$ and $q_{F}$. This is analogous to examining the asymptotic, flat spacetime limit of a spacetime geometry due to a source - gravity is evident close to the source, but hardly so, far from it. Similarly, there is a Minkowski-flat analog of the octonionic space, wherein the effect of $q_{B}$ and $q_{F}$ (which in effect 'curve' the octonionic space) is ignorable, and the asymptotic fine structure can be computed. The significance of the non-commutative, non-associative octonion algebra and the Jordan eigenvalues lies in that they already determine the coupling constants, including their asymptotic values. This is a property of the algebra, even though the interpretation of a particular constant as the fine structure constant comes from the dynamics, i.e. the Lagrangian, as it should, on physical grounds.

Quantum non-locality: Additional internal spatial dimensions which are not compact, yet very thin, offer a promising resolution to the quantum non-locality puzzle, thereby lifting the tension with 4D special relativity. Let us consider once again Baez's cube of Fig. 3. Any of 
the three quaternionic spaces containing the unit element 1 can play the role of the emergent 4D classical space-time in which classical systems evolve. Let us say this classical universe is the plane $\left(1 e_{6} e_{1} e_{5}\right)$. Now, the true universe is the full $8 \mathrm{D}$ octonionic universe, with the four internal dimensions being probed [only by] quantum systems. Now we must recall that these four internal dimensions are extremely thin, of the order of Fermi dimensions, and along these directions no point is too far from each other, even if their separation in the classical 4D quaternion plane is billions of light years! Consider then, that Alice at 1 and Bob at $e_{1}$ are doing space-like separated measurements on a quantum correlated pair. Whereas the event at $e_{1}$ is outside the light cone of 1 , the correlated pair is always within each other's quantum wavelength along the internal directions, say the path $\left(1 e_{3} e_{2} e_{7} e_{1}\right)$. The pair influences each other along this path acausally, because this route is outside the domain of 4D Lorentzian spacetime and its causal light-cone structure. The internal route is classically forbidden but allowed in quantum mechanics. This way neither special relativity nor quantum mechanics needs to be modified. It is also interesting to ask if evolution in Connes time in this 8D octonionic universe obeying generalised trace dynamics can violate the Tsirelson bound.

The exceptional Jordan algebra is of significance also in superstring theory, where it has been suggested that there is a relation between the EJA and the vertex operators of superstrings, and that the vertex operators represent couplings of strings [32, 33]. This intriguing connection between the EJA, string theory and aikyon theory deserves to be explored further.

Lastly we mention that the Lagrangian (45) that we have been studying closely resembles the Bateman oscillator [34] model, for which the Lagrangian is

$$
L=m \dot{x} \dot{y}+\gamma(x \dot{y}-\dot{x} y)-k x y
$$

I thank Partha Nandi for bringing this fact to my attention. Considering that the Bateman oscillator represents a double oscillator with relative opposite signs of energy for the two oscillators undergoing damping, it is important to understand the implications for our theory. In particular, could this imply a cancellation of zero point energies between bosonic and fermionic modes, thus annulling the cosmological constant? And also whether this damping is playing any possible role in generating matter-anti-matter asymmetry? 
Acknowledgements: I would like to thank Carlos Perelman for discussions and helpful correspondence, and for making me aware of the beautiful work of Dray and Manogue on the Jordan eigenvalue problem. I also thank Tanmoy Bhattacharya, Cohl Furey, Garrett Lisi, Roberto Onoforio and Robert Wilson for useful correspondence and discussions.

\section{REFERENCES}

[1] Geoffrey M. Dixon, Division algebras, octonions, quaternions, complex numbers and the algebraic design of physics (Kluwer, Dordrecht, 1994).

[2] C. H. Tze and F. Gursey, On the role of division, Jordan and related algebras in particle physics (World Scientific Publishing, 1996).

[3] Cohl Furey, "Standard model physics from an algebra? Ph. D. thesis, university of Waterloo," arXiv:1611.09182 [hep-th] (2015).

[4] Cohl Furey, "Three generations, two unbroken gauge symmetries, and one eight-dimensional algebra," Phys. Lett. B 785, 1984 (2018).

[5] Cohl Furey, " $S U(3)_{C} \times S U(2)_{L} \times U(1)_{Y}\left(\times U(1)_{X}\right)$ as a symmetry of division algebraic ladder operators," Euro. Phys. J. C 78, 375 (2018).

[6] J. Chisholm and R. Farwell, "Clifford geometric algebras: with applications to physics, mathematics and engineering," (Birkhauser, Boston, 1996 Ed. W. R. Baylis) p. 365.

[7] G. Trayling and W. Baylis, "A geometric basis for the standard-model gauge group," J. Phys. A: Math. Theor. 34, 3309 (2001).

[8] Michel Dubois-Violette, "Exceptional quantum geometry and particle physics," Nuclear Physics B 912, 426-449 (2016).

[9] Ivan Todorov, "Exceptional quantum algebra for the standard model of particle physics," Nucl. Phys. B 938, 751 arXiv:1808.08110 [hep-th] (2019), arXiv:1911.13124 [hep-th].

[10] Michel Dubois-Violette and Ivan Todorov, "Exceptional quantum geometry and particle physics II," Nucl. Phys. B 938, 751-761 arXiv:1808.08110 [hep-th] (2019), arXiv:1808.08110 [hep-th].

[11] Ivan Todorov and Svetla Drenska, "Octonions, exceptional Jordan algebra and the role of the group $F_{4}$ in particle physics," Adv. Appl. Clifford Algebras 28, 82 arXiv:1911.13124 [hep-th] 
(2018), arXiv:1805.06739 [hep-th].

[12] Ivan Todorov, "Jordan algebra approach to finite quantum geometry," in PoS, Vol. CORFU2019 (2020 arXiv:) p. 163.

[13] Rafal Ablamowicz, "Construction of spinors via Witt decomposition and primitive idempotents: A review," in Clifford algebras and spinor structures, edited by Rafal Ablamowicz and P. Lounesto (Kluwer Acad. Publ., 1995) p. 113.

[14] John C. Baez, "The octonions," Bull.Am.Math.Soc. 39 (2002), arXiv:math/0105155 [math.RA].

[15] John C. Baez, "Division algebras and quantum theory," Foundations of Physics 42, 819-855 (2011).

[16] John C. Baez and John Huerta, "The algebra of grand unified theories," (2009 arXiv:0904.1556 [hep-th]), arXiv:0904.1556 [hep-th].

[17] Carlos Castro Perelman, " $R \times C \times H \times O$ valued gravity as a grand unified field theory," Advances in Applied Clifford Algebras 29, 22 (2019).

[18] Adam B. Gillard and Niels G. Gresnigt, "Three fermion generations with two unbroken gauge symmetries from the complex sedenions," The European Physical Journal C 79 (2019 arXiv:1904.03186), 10.1140/epjc/s10052-019-6967-1.

[19] Ovidiu Cristinel Stoica, "The standard model algebra (Leptons, quarks and gauge from the complex algebra $\mathrm{Cl}(6))$," Advances in Applied Clifford Algebras arXiv:1702.04336, 52 (2018).

[20] Ichiro Yokota, "Exceptional Lie groups," arXiv:0902.043 [math.DG] (2009).

[21] Tevian Dray and Corinne Manogue, "The exceptional Jordan eigenvalue problem," Int. J. Theo. Phys. 28, 2901 arXiv:math-ph/9910004v2 (1999).

[22] Tevian Dray and Corinne Manogue, "Octonions, $\mathrm{E}_{6}$ and particle physics," J.Phys.Conf.Ser. 254, 012005 arXiv:0911.2253 (2010).

[23] A. Garrett Lisi, "An exceptionally simple theory of everything," (2007), arXiv:0711.0770 [hep-th].

[24] Tejinder P. Singh, "Trace dynamics and division algebras: towards quantum gravity and unification." Zeitschrift für Naturforschung A arXiv:2009.05574v44 [hep-th], DOI: https://doi.org/10.1515/zna-2020-0255 (2020).

[25] Adam B. Gillard and Niels Gresnigt, "The $\mathrm{Cl}(8)$ algebra of three fermion generations with 
spin and full internal symmetries," arXiv:1906.05102 (2019).

[26] A. Adrien Albert, "On a certain algebra of quantum mechanics," Annals of Mathematics 35, 65 (1933).

[27] P. Jordan, John von Neumann, and E. Wigner, "On an algebraic generalisation of the quantum mechanical formalism," Ann. Math. 35, 65 (1933).

[28] K. A. Olive; et al. (Particle Data Group) (2014), "Review of particle properties," Chinese Physics C. 38 (9) 38, 1 (2014).

[29] Tejinder P. Singh, "Quantum gravity, minimum length and hologaphy," to appear in Pramana - J. Phys. arXiv:1910.06350 (2020).

[30] Maithresh Palemkota and Tejinder P. Singh, "Proposal for a new quantum theory of gravity III: Equations for quantum gravity, and the origin of spontaneous localisation," Zeitschrift für Naturforschung A 75, 143 (2019 DOI:10.1515/zna-2019-0267 arXiv:1908.04309).

[31] Roberto Onofrio, "High energy density implications of a gravitoweak unification scenario," Mod. Phy. Letts. A 29, 1350187 (2014).

[32] E. Corrigan and T. J. Hollowood, "Exceptional jordan algebra and the superstring," Commun. Math. Phys. 122, 393 (1989).

[33] P. Goddard, W. Nahm, D. Olive, H. Ruegg, and A. Schwimmer, "Fermions and octonions," Commun. Math. Phys. 112, 385 (1987).

[34] Kunihiko Nakano Shinichi Deguchi, Yuki Fujiwara, "Two quantization approaches to the Bateman oscillator model," Ann. Physics 403, 34 arXiv:1807.04403 [quant-ph] (2019). 